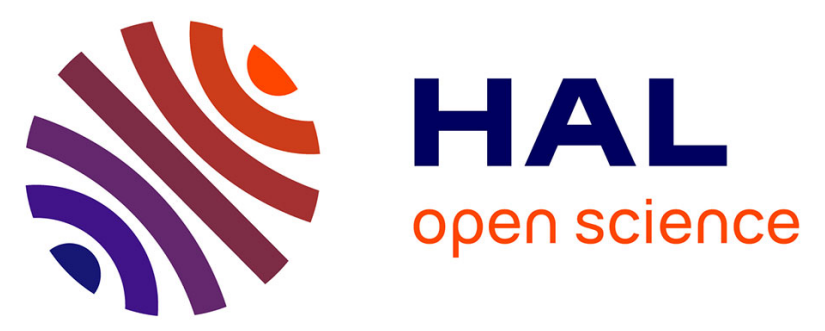

\title{
Un simulateur pour répondre à des besoins de formation sur la taille de la vigne
}

\author{
Sylvie Caens-Martin, Antonietta Specogna, Ludovic Delépine, Stéphane
}

Girerd

\section{- To cite this version:}

Sylvie Caens-Martin, Antonietta Specogna, Ludovic Delépine, Stéphane Girerd. Un simulateur pour répondre à des besoins de formation sur la taille de la vigne. STICEF (Sciences et Technologies de l'Information et de la Communication pour l'Éducation et la Formation), 2004, 11, 13 p. hal-00696442

\section{HAL Id: hal-00696442 \\ https://hal.science/hal-00696442}

Submitted on 11 May 2012

HAL is a multi-disciplinary open access archive for the deposit and dissemination of scientific research documents, whether they are published or not. The documents may come from teaching and research institutions in France or abroad, or from public or private research centers.
L'archive ouverte pluridisciplinaire HAL, est destinée au dépôt et à la diffusion de documents scientifiques de niveau recherche, publiés ou non, émanant des établissements d'enseignement et de recherche français ou étrangers, des laboratoires publics ou privés. 


\section{Un simulateur pour répondre à des besoins de formation sur la taille de la vigne}

Sylvie Caens-Martin, Antonietta Specogna Etablissement National de l'Enseignement Supérieur Agricole de Dijon (ENESAD) ]

Ludovic Delépine, Stéphane Girerd [Centre National d'Etudes et de Ressources en Technologies Avancées (CNERTA)]

n RÉSUMÉ : Notre article a pour objectif de présenter un simulateur de taille de la vigne conçu pour servir d'instrument dans une situation de formation où sont impliqués un formateur, le simulateur et des apprenants. L'objectif de ce simulateur est de permettre à un apprenant d'acquérir des raisonnements de taille tels que développés par un tailleur professionnel sur le terrain. Il ne porte pas sur l'apprentissage des gestes de taille mais sur la construction des raisonnements participants aux compétences visées. Nous nous efforcerons au fur et à mesure de cet article de montrer que la conception et la réalisation de cette situation de formation dans sa globalité font intervenir des compétences multiples qu'aucune des disciplines impliquées (didactique professionnelle, psychologie, informatique) prises indépendamment ne peut fournir à elle seule.

MOTS CLÉS : Simulation, formation, évaluation, taille de la vigne.

- Introduction

- Quels sont les éléments sur lesquels s'appuyer à partir d'une analyse du travail en didactique professionnelle?

- Une situation de formation tutorée appareillée

- Une présentation générale de l'architecture du simulateur de taille

- Une situation de taille de la vigne en Guyot dans le simulateur

- Le Processus de conception du simulateur : le travail d'une équipe pluridisciplinaire.

- Des situations d'observation du simulateur en situation

- Conclusion

- $\underline{\text { Références }}$

\section{Introduction}

Une maîtrise des quotas de production, de nouvelles exigences de qualité, encouragées par la reconnaissance des vins en $A O C$ et le développement du caractère régional de la commercialisation des vins de terroir amènent la profession viticole à reconsidérer les pratiques de conduite du vignoble et notamment celle de la taille. A ce constat s'ajoute le fait que la population actuelle de tailleurs est relativement âgée et qu'il y aura nécessité de former les plus jeunes dans les années prochaines. Les nouvelles données économiques et la nécessité d'avoir de "bons tailleurs" ont amené la profession à se tourner vers les acteurs de la formation en vue de concevoir des modalités de formations permettant de construire et d'améliorer les compétences de taille pour cette main d'œuvre. 
Cette demande, a été relayée par le FAFSEA (Fonds national d'Assurance Formation des Salariés des Exploitations et Entreprises Agricoles) auprès de l'ENESAD : Etablissement National d'Enseignement Supérieur Agronomique de Dijon (Équipe de Didactique Professionnelle). Deux Contrats de Plan État Région, ont permis d'associer à ce projet le FAFSEA, le CNERTA-ENESAD (Centre National d'Etudes et de Ressources en Technologies Avancées) et le LEAD/CNRS UMR 5022 Université de Bourgogne (Laboratoire d'Étude de l'apprentissage et du Développement).

Nous présentons tout d'abord les éléments issus d'une analyse du travail en didactique professionnelle sur lesquelles la conception d'un simulateur de taille de la vigne peut s'appuyer. Puis, nous présentons la situation de formation tutorée appareillée d'un simulateur utilisé comme un instrument technologique support pour l'apprentissage d'un raisonnement. Ensuite, nous explicitons l'architecture générale du simulateur de taille de la vigne. À la suite de cette présentation, nous montrons une situation de taille dans un prototype de simulateur réalisé et spécifié pour la taille de la vigne en Guyot. Enfin, nous présentons quelques résultats obtenus lors de la réalisation de situations d'observation autour de ce simulateur et dont les résultats sont réinvestis dans un simulateur en cours de réalisation.

\section{Quels sont les éléments sur lesquels s'appuyer à partir d'une analyse du travail en didactique professionnelle?}

L'analyse du travail en didactique professionnelle, comme l'exposent [Pastré97], [Mayen01] et [SauretGuibert03] se décompose en trois temps :

- La connaissance du domaine inscrite dans une logique d'exposition qui relève de la compréhension de l'objet sujet à des transformations au moyen de l'analyse de données scientifiques et techniques, ainsi que de la prescription de la tâche et des caractéristiques proches de la tâche (buts, moyens et contraintes dans l'organisation);

- La connaissance du domaine inscrite dans une logique d'action en situation par l'observation du travail effectif des professionnels experts. Cette étude s'appuie sur l'analyse des gestes et des raisonnements qui s'inscrivent dans la diversité et la variabilité des situations rencontrées ;

- L'identification des écarts entre les compétences mobilisées dans la situation professionnelle de référence (situation construite à partir des deux précédents points) et les compétences en construction effectivement mobilisées dans les situations d'apprentissage existantes.

Dans le contexte spécifique de la taille de la vigne, nous allons présenter comment se décrivent ces principes.

\subsection{La logique d'exposition}

L'objet étudié : le cep de vigne est une plante vivante pérenne d'une durée de vie approximative de cinquante ans. Cette plante possède des mécanismes d'autorégulation biologiques que le tailleur peut orienter à son profit [CaensMartin99] dans une perspective de production dans un environnement mécanisable.

Le but : l'objet du travail de taille consiste à produire régulièrement du raisin sans engager le capital vie de la plante dans un contexte législatif précis.

\subsection{La logique d'action et les difficultés d'apprentissage}

L'activité de taille de la vigne est très particulière dans le domaine agricole [CaensMartin99] car pour être effectuée correctement, un tailleur doit prendre en compte des critères tels que la position relative du cep 
dans l'espace, la qualité des bois de souche, la vigueur de la souche ou le réseau de distribution de la sève pour lesquels il n'existe pas d'éléments de mesure sur lesquels s'appuyer a priori.

Avant toute chose, un tailleur expert doit impérativement effectuer sa taille du moment dans une perspective à long terme. Tailler une vigne en oubliant cette perspective pourrait être absolument néfaste pour celle-ci car si la taille n'est pas effectuée correctement une année alors cela risque de provoquer une irrégularité dans le développement du plan de vigne et dans sa production. Ce point soulève pour l'apprenant une première difficulté de regard qui est d'inscrire son diagnostic de la situation et son action dans un contexte temporel beaucoup plus large que celle de l'intervention immédiate ("ici et maintenant"). Cette difficulté de contrôle des décisions de taille est renforcée par le fait que l'apprenant n'aura pas l'occasion de revenir sur la parcelle dans laquelle il a effectué ses tailles aux moments du développement de la vigne, de la vendange et de la taille l'année suivante.

A partir d'un recueil de données pragmatiques, l'analyse du travail a permis d'identifier les concepts mobilisés dans et pour une action efficace. Deux concepts organisent l'activité de diagnostic et d'action : le concept de charge et le concept d'équilibre.

La charge est le calcul que le tailleur doit faire pour attribuer au cep le nombre d'yeux fructifères en fonction de sa vigueur.

Le concept d'équilibre s'observe lui-même sous deux angles : l'architecture du cep et les réseaux de distribution de la sève. L'équilibre architectural renvoie à la forme du cep, son orientation et son développement par rapport à l'axe du rang. L'équilibre du réseau de distribution de la sève concerne l'alimentation proportionnelle de l'ensemble des organes du cep.

La variabilité des situations de taille amène le professionnel à mobiliser systématiquement ces deux concepts dans des proportions différentes. Ce point est une seconde difficulté pour l'apprenant qui a tendance à privilégier l'un des deux concepts, généralement celui de charge. Les situations d'apprentissage sur un rang de vigne réel ne permettent pas d'apprendre à construire l'utilisation de ces deux concepts. L'apprenant et le formateur n'ont pas toujours la possibilité de rencontrer des cas de taille qui pourraient permettre de distinguer l'influence de ces deux concepts et donc la mise en évidence d'alternatives de taille.

De plus, cette analyse du travail met en évidence le fait que quelle que soit la conduite de taille utilisée (en Guyot simple ou double, en Cordon de Royat ou en Gobelet), ces deux concepts sont toujours mobilisés. Ils permettent grâce aux variables construites (qualité des bois, vigueur, position dans l'espace, réseau de distribution de sève), et donc aux indicateurs prélevés pour qualifier voir quantifier ces variables, de porter un diagnostic de la situation de taille et de contrôler la variabilité des situations rencontrées [CaensMartin00].

Enfin, nous pouvons préciser que cette taille représente une activité physique difficile car elle s'opère courbée sur de grandes surfaces pendant l'hiver, période où les conditions climatiques rendent ce travail, parfois saisonnier, laborieux.

\section{Une situation de formation tutorée appareillée}

Le point de vue pris pour la conception du simulateur tel qu'il existe présente un changement radical par rapport à d'autres produits multimédias d'aide à l'apprentissage. Ce simulateur entre délibérément par l'apprentissage des situations de travail. En cela, il n'est ni un exerciseur à proprement parler, ni un système expert, il impose l'intervention d'un formateur [CaensMartin02].

La conception du simulateur s'appuie sur une série de travaux de recherches menés sur l'utilisation et le rôle en formation professionnelle des outils de simulation : la conduite des hauts fourneaux, de presses à injecter, de centrales nucléaires, le pilotage d'avion, la gestion des feux de forêt, la conduite de grues 
([Leplat89], [Pastré92], [RogalskiSamurçay94], [SamurçayRogalski98], [Boucheix03]). Le simulateur part des situations de travail et sa construction se situe dans une logique de médiation entre une situation de référence et un sujet apprenant, via une série de situations simulées.

En utilisant la définition qu'en donnent Samurçay et Rogalski ([RogalskiSamurçay94], [SamurçayRogalski98]), "on appelle situation de référence la classe de situations de travail qui, dans un processus de formation, est la cible du développement de la compétence". Pour nous, la situation de référence a été modélisée et validée par les experts professionnels de la taille de la vigne, elle concerne l'environnement à gérer et l'activité attendue.

Les situations simulées nécessitent, quant à elles, d'opérer des choix didactiques, une transposition didactique. Ce point de vue amène d'une part à produire des scénarii pour gérer la complexité des apprentissages par décomposition puis recomposition des connaissances et des compétences, d'autre part à concevoir un modèle d'environnement qui conserve les fonctionnalités des situations de références et qui prenne en compte les difficultés d'apprentissage repérées chez les apprenants.

La transposition ([Chevalard85], [SamurçayRogalski98]) à effectuer doit mettre en scène et prendre en compte :

- la conception de situations d'apprentissage qui permet la construction des composantes des compétences cibles pour cette classe de situations professionnelles,

- le niveau de complexité de la situation simulée,

- un environnement d'apprentissage qui met à disposition de l'apprenant les outils d'investigation et de contrôle qu'utilise le professionnel dans ses raisonnements en situation réelle,

- les objets à transformer dans un environnement vraisemblable,

- et, bien évidemment, le sujet apprenant et ses difficultés d'apprentissage.

Dans notre contexte, les intentions didactiques du concepteur liées à cette transposition et qui organisent l'environnement d'apprentissage du simulateur de taille sont les suivantes :

- garder de la situation les caractéristiques principales pour la construction des compétences ;

- décomposer les apprentissages en construisant des classes de situations de taille pour un apprentissage et un étayage des concepts à maîtriser et des règles à mobiliser ;

- compresser le temps de réponse du végétal à la durée de la session, ce qui permet à l'utilisateur de visualiser l'effet de ses choix sur l'évolution de l'architecture du cep ;

- offrir à l'utilisateur la possibilité de proposer plusieurs solutions de taille et de les visualiser ;

- exploiter a posteriori les traces de l'action entreprise pour résoudre la situation en suivant l'historique des investigations menées et comprendre les raisonnements et les règles d'actions utilisées ;

- proposer une même situation ou une même classe de situations à plusieurs apprenants ;

- créer de nouvelles situations d'apprentissage autres que celles déjà proposées par défaut dans le simulateur à partir de l'utilisation d'une "banque d'organes" mise à la disposition du formateur.

Comme nous l'indiquons, le simulateur s'appuie sur les situations de travail transposées en situations d'apprentissage. La gestion d'un environnement dynamique vivant, tel que le cep de vigne, ne permet pas d'introduire dans le dispositif une validation de l'action comme bonne ou mauvaise, l'organisation de prises d'informations comme adéquate à la situation ou non, les choix de la décision de taille comme la plus opérante ou non compte tenu de la situation de départ. Le simulateur n'est non plus pas conçu pour faire le lien entre des choix d'action et un système d'expertises qui permettrait de faire référence aux connaissances scientifiques et techniques qui éclairent l'action entreprise. Même si ce simulateur met à la disposition de l'apprenant les outils d'investigation pour comprendre la situation, il ne donne pas une aide à la mise en liaison entre structures, fonctions et comportement de l'objet transformé. Enfin, le simulateur ne permet pas de donner les outils pour créer des liens de causalité entre variables. Ce manque implique alors un dispositif de formation où l'intervention immédiate et/ou différée d'un formateur technique, 
selon la classification de Gravé [Gravé03], dans le processus de construction des compétences est nécessaire. Le dispositif ainsi conçu se place dans la vision de Samurcay qui montre les rapports des trois pôles que sont l'apprenant, le formateur et l'outil de simulation en lien avec la situation professionnelle de référence cible du développement des compétences [Samurçay00].

Il s'avère alors au regard de ces éléments qu'une situation de formation tutorée avec un binôme technohumain formateur-simulateur semble être la situation de formation la plus adaptée à satisfaire l'objectif visé : améliorer l'apprentissage de la taille de la vigne, en espérant obtenir ensuite une meilleure qualité de taille et donc une meilleure récolte.

Pour toutes ces raisons, la décision de réaliser un simulateur de taille de vigne a été prise car la simulation permet d'approximer un environnement complexe :

- pour présenter des situations contrôlées dans lesquelles des opérations délicates peuvent être réalisées sans risques ;

- pour fournir aux apprenants un moyen de contrôle sur une situation et leur fournir des explications appropriées ;

- pour simplifier la situation réelle et ne présenter que les éléments saillants relativement à l'objectif de la formation, compte tenu des connaissances scientifiques du domaine concerné par ailleurs.

\section{Une présentation générale de l'architecture du simulateur de taille}

Le choix a été fait d'éviter de concevoir un instrument de formation fermé mais bien au contraire d'offrir de larges possibilités d'utilisation tant du côté de l'apprenant, confronté à des situations de taille à résoudre et à la possibilité d'analyser après coup son travail, que du côté du formateur qui peut créer luimême les situations de taille à résoudre et suivre les apprentissages des apprenants. Ainsi le simulateur est composé de trois modules que nous allons présenter: les situations de taille à résoudre (module exercices), l'historique et la création d'exercice.

La mise en scène des situations d'apprentissage concerne en premier lieu le module des situations de taille à résoudre, module central dans le simulateur.

- Le module possède les fonctionnalités qui permettent à l'apprenant comme au professionnel sur le terrain de prendre les informations pertinentes de la situation à partir desquelles il portera un diagnostic de la situation qui lui est proposée et décidera de ses choix de taille.

- Une modélisation de la croissance des bois permet de visualiser les effets de la taille à l'année suivante où la compression du temps de croissance des bois permet le contrôle de l'action.

- Cette nouvelle situation de taille, créée par l'apprenant à partir d'une situation de départ imposée, peut être immédiatement reprise sur trois années consécutives et ainsi permettre de conduire un raisonnement de suivi de l'architecture et de la vigueur d'un même cep sur plusieurs années.

- Les situations à résoudre sont regroupées de telle sorte qu'elles permettent à l'apprenant de construire les concepts, les variables et les indicateurs qui fondent les raisonnements des professionnels.

Le but de ce module est de créer les conditions d'un développement des compétences "en situation" pour un apprentissage par l'exercice de l'activité.

Si on considère avec Pastré [Pastré97] que la construction des compétences se fait par apprentissage des situations de travail mais aussi par l'analyse après coup de son travail, d'un point de vue conception didactique, le simulateur doit posséder des fonctionnalités qui permettent d'accéder facilement au repérage des raisonnements, qui ont conduit l'apprenant à la recherche d'informations et à l'action, c'est la réalisation du module historique. Par une fonction de marquage des différentes actions menées par 
l'apprenant dans le module exercices, ce module permet à l'apprenant comme au formateur de suivre et d'analyser "après coup" la succession des opérations réalisées pour résoudre chaque exercice. Le but de ce module est de créer les conditions d'une activité de retour sur l'action de l'apprenant : "ce que j'ai fait, pourquoi je l'ai fait". Il a aussi pour le formateur une fonction d'évaluation.

Le formateur dispose des outils nécessaires à la création de nouvelles situations d'apprentissage qu'il peut inclure dans son dispositif de formation et la gestion des situations d'apprentissage, c'est la réalisation du module création d'exercices. Ce module permet à un formateur de modifier des exercices déjà existants ou d'en concevoir de nouveaux à partir d'une banque d'organes nécessaires à la construction du cep, d'intégrer des maladies sur les bois, des accidents mécaniques ou climatiques par exemple. Chaque exercice est accompagné d'une consigne de travail adressée à l'apprenant. Le but de ce module est de permettre au formateur de créer les situations d'apprentissage propices au développement des compétences, en fonction de sa progression de formation et du niveau du public d'apprenants.

\section{Une situation de taille de la vigne en Guyot dans le simulateur}

Nous avons choisi de présenter plus particulièrement l'usage du module exercice par un apprenant. Ainsi, lors d'une session de travail sur le simulateur, l'apprenant a deux modes de travail, un mode guidé et un mode libre. Dans le mode guidé, l'apprenant suit un chemin de travail préalablement construit qui l'amène à passer par toutes les étapes de prises d'informations et de diagnostic nécessaire à la taille l'exercice.

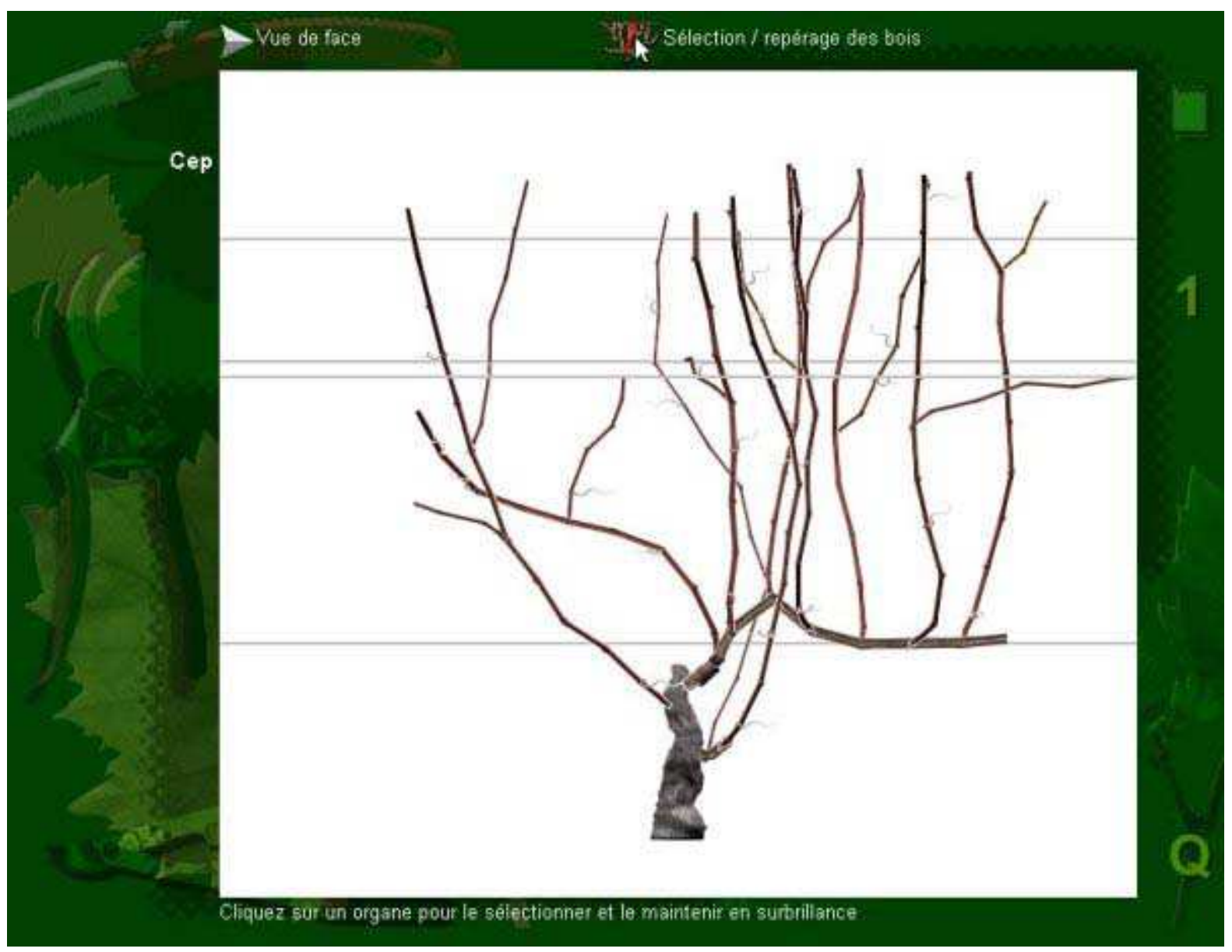

Figure 1 : La représentation 2D d'un cep en vue de côté. 
Le mode libre permet à l'apprenant de choisir lui-même la succession des opérations à réaliser pour résoudre

Avant de démarrer un exercice, dans l'un ou l'autre des deux modes, l'apprenant doit s'identifier puis il choisit un des exercices proposés par le formateur. Un écran présente alors la consigne de taille de l'exercice. Puis, le cep s'affiche dans la partie centrale de l'écran.

Dans un mode libre, l'apprenant peut effectuer les opérations qu'il juge utiles et dans l'ordre dans lequel il le souhaite. Il peut effectuer des observations sur le cep au moyen d'une "loupe" en la plaçant sur une partie de celui-ci. Il peut alors demander des informations au simulateur :

- $\quad$ sur les bois présentés (le type de bois et d'organe, son orientation),

- sur les bourgeons laissés l'année précédant l'année de taille en cours simulée, sur les maladies et les accidents (gel, grêle par exemple),

- sur la qualité des sarments (empattement, aoûtement, diamètre des bois),

- sur une évaluation de la vigueur et de la charge du cep.

Il peut de même observer le cep avec une vue de côté ou une vue de dessus. Ensuite, l'apprenant peut sélectionner les futurs coursons et baguettes sur le cep présenté. Il peut tailler le cep au moyen de divers instruments (sécateur, sécateur à démonter ou scie). Enfin, il peut ébourgeonner et attacher la baguette retenue dans la direction choisie (à droite ou à gauche). L'ensemble des opérations effectuées par l'apprenant sur le cep provoque un calcul en temps réel de la représentation du nouveau cep obtenu. Ces activités peuvent être réalisées à cinq reprises sur le même cep et en parallèle par l'apprenant pour obtenir des ceps taillés candidats à une simulation de croissance.

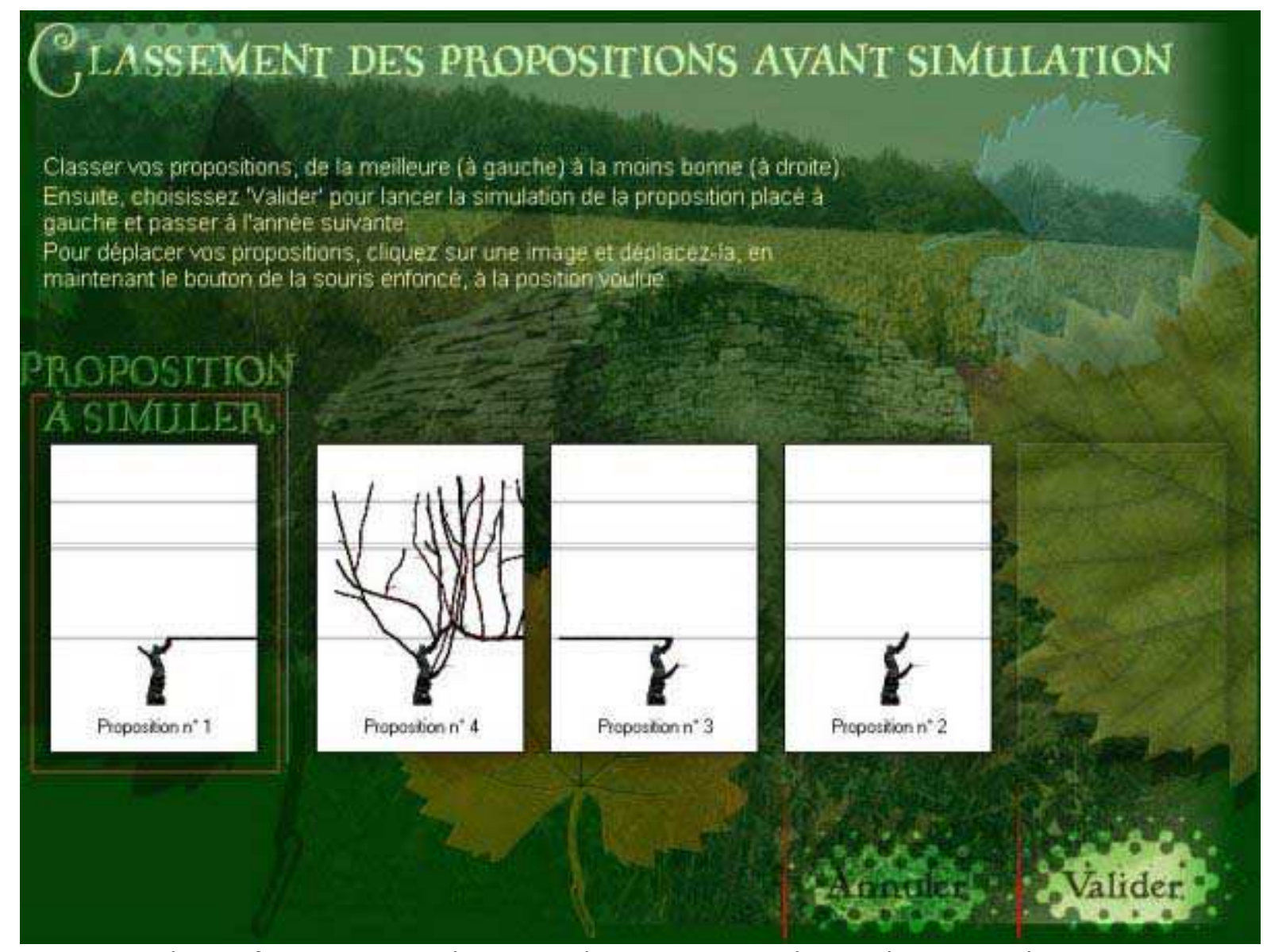

Figure 2 : Des ceps taillés candidats avant la simulation de croissance. 
Lorsque celui-ci estime qu'il a réalisé suffisamment de candidats, il peut en chosir un et simuler sa croissance sur une année. Il peut ensuite rééffectuer un processus de taille sur le nouveau cep obtenu.

A tout moment, l'apprenant dispose d'un calepin électronique qui lui permet de laisser des traces de son activité.

\section{Le Processus de conception du simulateur : le travail d'une équipe pluridisciplinaire.}

Passer des intentions didactiques à la réalisation informatique du simulateur nécessite la collaboration de personnes issues d'horizons de travail très différents. Pour construire les bases d'une inter compréhension entre le "monde de la formation" et celui de l'informatique un synopsis a été réalisé par le concepteur. Ce travail a permis d'identifier les liens entre les intentions du concepteur et ce qu'il voulait permettre à l'utilisateur-apprenant de faire et dans quelles conditions d'activation de l'action.

La première étape du travail collectif a abouti à la production d'un cahier des charges de la programmation. Le développement proprement dit a utilisé les outils suivants: VISUAL BASIC et ACCES pour l'interface, la base de données, et le moteur de croissance des bois et 3D STUDIO MAX pour la réalisation graphique des organes du cep.

$\mathrm{Au}$ delà du cahier des charges, la collaboration pluridisciplinaire a été maintenue et c'est une caractéristique de ce projet. Elle a permis des réajustements successifs tout au long des étapes de réalisation informatique et jusqu'à la production finale du prototype.

Pour l'équipe qui a collaboré à cette production les difficultés rencontrées ont essentiellement résidé dans la construction d'un langage permettant une compréhension réciproque des "besoins" et des "possibles". Pour cela une confrontation des points de vue a permis de faire émerger le sens à donner au travail de production qui était de mettre en place un langage commun pour parler du travail de taille, et de l'objet cep (ses caractéristiques morphologiques et physiologiques) et de traduire en langage de l'informatique les intentions didactiques initiales.

\section{Des situations d'observation du simulateur en situation}

"Lorsqu'un système est trop complexe pour être compris, spécifié ou réalisé du premier coup, voire les trois à la fois, il vaut mieux le réaliser en plusieurs fois par évolution" [Gall86]. Ainsi, comme Gall le fait remarquer de manière générale, la conception et la réalisation de ce type d'instrument logiciel complexe ne peuvent faire l'économie d'un développement itératif où chaque fin d'itération est marquée par une étape d'évaluation scientifiquement validée. Dans ce contexte, des situations d'observation du simulateur ont été mises en place par les informaticiens, les didacticiens, les psychologues et les experts métiers. Ces situations portent sur :

- les fonctionnalités proposées par le simulateur (Bodillard, Boucheix, Caens-Martin) ;

- une observation d'une situation de formation simulateur-apprenant (Condémine, Boucheix, CaensMartin [Condemine03]) ;

- l'introduction du simulateur dans un dispositif de formation formateur-simulateur-apprenant (Barry, Caens-Martin).

Dans ces situations, l'appropriation du simulateur par les formateurs et les apprenants a permis de faire un grand nombre de remarques visant à améliorer le prototype du simulateur. Les catégories de commentaires sont les suivantes : 
- pédagogique : le mode d'utilisation guidé du simulateur qui oblige à analyser tous les bois du cep est jugé long et inutile. Il est proposé de le supprimer. De même, les apprenants souhaitent connaître le niveau de difficulté de la taille d'un cep avant de la commencer et non pas de disposer d'une liste de cas sans informations a priori;

- fonctionnelle : des fonctionnalités supplémentaires sont souhaitées telles que pouvoir revenir en arrière lorsqu'une erreur de taille est commise suite à un problème de maniement du curseur afin d'éviter de recommencer une taille complète, la simulation de l'ensemble des candidats retenus par l'apprenant pour comparer les évolutions liées au choix de taille ou encore la possibilité de simuler l'ébourgeonnage printanier comme étape en tant que telle dans la simulation de pousse du cep ;

- dispositionnelle : des évolutions afin de rendre plus facilement accessibles les opérations possibles à effectuer sur un cep ainsi que leurs rôles dans la taille ont été précisées ;

- graphique: les apprenants et les formateurs ont souhaité une évolution de la représentation bidimensionnelle à une représentation tridimensionnelle du cep afin de mieux rendre compte des positions relatives des bois dans l'espace, la possibilité d'utiliser une loupe afin de mieux se rendre compte d'informations accessibles visuellement sur le cep, l'augmentation et la diversification des éléments d'architecture utilisés pour préparer les instances de ceps contenues dans la base de cas ainsi qu'une représentation plus réaliste des maladies sur les bois ;

- didactique : les apprenants et les formateurs souhaitent distinguer la consigne de travail donnée à l'apprenant pour traiter l'exercice de taille, des ordres de taille donnés dans une exploitation sur la parcelle auquel appartiendrait le cep objet de l'exercice.

L'ensemble de ces commentaires recueillis sert de base à la conception d'un simulateur qui est actuellement en cours de réalisation. Ce simulateur, totalement réécrit, bénéficie d'une architecture technique radicalement différente du premier prototype afin d'en renforcer la stabilité et d'en faciliter l'évolutivité. Il profite également de l'ensemble des technologies nouvellement accessibles pour la représentation tridimensionnelle de formes. Une nouvelle évaluation et une validation pluridisciplinaire de ce prototype sont de même prévues à la fin de cette étape de développement qui devrait, selon les prévisions respectives des protagonistes de ce projet, être quasi-terminale. Cela permettrait ainsi d'assurer la convergence du développement d'un simulateur réalisé au sein d'un développement itératif.

\section{Conclusion}

Cet article a montré que la conception et la réalisation d'un tel simulateur de taille de la vigne n'ont été rendues possibles qu'à la suite d'une analyse du travail de la taille très précise. Il est ensuite devenu le fruit d'une collaboration pluridisciplinaire entre la didactique professionnelle, la psychologie, les informaticiens en association avec les professionnels du secteur viticole.

Ce prototype de simulateur a suscité de la part des professionnels des régions Aquitaine, ChampagneArdenne, Bourgogne et Languedoc-Roussillon un grand intérêt. Relayé en cela par le FAFSEA et financé pour partie par des fonds européens, un nouveau simulateur de taille de la vigne a été confié à l'ENESAD. Ce nouvel outil de formation concerne d'autres types de taille de production mais aussi de taille de formation de jeunes ceps (Guyot, Guyot double, Cordon de Royat, Chablis, Gobelet) ainsi que d'autres types de cépages propres à certaines régions viticoles. De plus, il inclut une simulation des interventions en vert complémentaire à la taille d'hiver déjà simulée.

Traditionnellement, l'objectif de l'apprentissage par simulateur est de pouvoir donner la possibilité à l'apprenant de construire de l'expérience pour la gestion de systèmes complexes et le traitement efficace de systèmes de routine lorsque l'apprentissage par essai/erreur peut être dangereux, et donc non toléré. La particularité de ce simulateur est d'associer dans une situation de formation un formateur et un simulateur de sorte qu'il nous semble important de considérer l'introduction de ce simulateur comme un instrument 
pour enseigner et pour apprendre, au sens de Rabardel [Rabarde197], et non pas comme un gadget technologique se substituant au monde.

\section{Références}

\section{BIBLIOGRAPHIE}

\section{[Boucheix03]}

Boucheix, J.M. Simulation et compréhension de documents techniques: le cas de la formation des grutiers. Le travail humain, à paraître, 2003.

\section{[Caens-Martin99]}

Caens-Martin, S. Une approche de la structure conceptuelle d'une activité agricole : la taille de la vigne. Education permanente, No. 139, 1999, pp. 99-114.

\section{[Caens-Martin00]}

Caens-Martin, S. Former à la conceptualisation des situations professionnelles. Le cas de la taille de la vigne. Rapport de recherche No. 98511277, ENESAD Équipe de Didactique Professionnelle, 2000.

\section{[Caens-Martin02]}

Caens-Martin, S. Conception, réalisation, et évaluation d'un outil multimédia de formation à la taille. Rapport de recherche, Contrat de plan Etat-Région : Recherche et transfert de technologie, ENESAD Équipe de Didactique Professionnelle, 2002.

\section{[Chevalard85]}

Chevalard, Y. La transposition didactique. Grenoble, 1985.

\section{[Condemine03]}

Condemine, L. Expérimentation du simulateur taille de la vigne en Guyot. DESS d'Ingénierie des Apprentissages en Formation Professionnelle, Université de Bourgogne et ENESAD, janvier 2003.

\section{[Gravé03]}

Gravé, P. L'identité professionnelle des formateurs. Sciences humaines hors série No. 40, Former, se former, se transformer, Mars-avril-mai 2003, pp. 38-41.

\section{[Gall86]}

Gall, J. How System Really Work and How They Fail. Ann Arbor (Eds), The General systematics Press, 1986.

\section{[Lecoq98]}

Lecoq, R. Viticulture : la taille de l'hiver joue un rôle d'arbitre. VITI, No. 226, 1998.

\section{[Leplat89]}

Leplat, J. Simulation and simulator in training : some comments. dans Brainbridge, L., Quintanilla, R. (Eds), Developng skill with information technology, Chichester, Wiley and Sons Eds, 1989.

\section{[Mayen01]}

Mayen P. Développement professionnel et formation : une théorie didactique. Thèse pour l'Habilitation à Diriger des Recherches en Sciences de l'Education, Université Pierre Mendes-France, Grenoble, 2001. 


\section{[Pastré92]}

Pastré, P. Essai pour introduire le concept de didactique professionnelle. Rôle de la conceptualisation dans la conduite de machines automatisées. Thèse de Doctorat, Université de Paris 5, 1992.

[Pastré97]

Pastré, P. Didactique professionnelle et développement. Psychologie Française, No. 42, 1997.

\section{[Rabardel97]}

Rabardel, P. Activités avec instruments et dynamique cognitive du sujet. Dans Moro, C., Schneuwly, B., Brossard, M. Outils et signes, Peterlang, Berne, 1997.

\section{[RogalskiSamurçay94]}

Rogalski, J. Samurçay, R. Modélisation d'un savoir de référence et transposition didactique dans la formation de professionnels de haut niveau. Dans Arsac, G., Chevallard, Y., Martinand, J.L., Tiberghien, A., La transposition didactique à l'épreuve, La pensée sauvage, Grenoble, 1994.

\section{[SamurçayRogalsky98]}

Samurçay, R., Rogalsky, J. Exploitation didactique des situations de simulation. Le travail humain, Vol. 61, No. 4, Décembre 1998, pp. 333-360.

\section{[Samurçay00]}

Samurçay, R. Concevoir des situations simulées pour la formation professionnelle: une approche didactique. Document de travail présenté au séminaire de Didactique Professionnel de l'ENESAD, 2000.

\section{[Sauret-Guibert03]}

Sauret-Guibert, S. Les enjeux de la conception et de l'usage d'une ressource éducative pour la transmission des savoir-faire en entreprise. Le cas d'un simulateur didactique pour les lamineurs à chaud. Thèse de doctorat en sciences de l'éducation. Université Parsi XIII, 2003.

\section{Equipe de conception et de realisation du prototype}

Sylvie Caens-Martin : Équipe de recherche en Didactique professionnelle, ENESAD, responsable de la recherche.

Serge Monnet, ingénieur de conception et responsable de la conception et du développement Stéphane Girerd, Rodolphe Pellerin (infographiste 3D) : Équipe de conception multimédia pédagogique, ENESADCNERTA.

Joël Durier : expert spécialiste de la taille et formateur au Lycée Viticole de Beaune.

Sous l'égide du FAFSEA, les professionnels viticoles des régions Languedoc-Roussillon et Bourgogne.

\section{- A propos des auteurs}

- Sylvie CAENS-MARTIN, assistant ingénieur, est chercheur associé dans l'UP Développement Professionnel et Formation, équipe de Didactique Professionnelle à l'ENESAD de Dijon. Ses travaux de recherche visent à comprendre le travail de professionnels et les difficultés d'apprentissage en vue de produire des ressources pour la formation. Ses thèmes d'étude concernent - L'analyse des compétences professionnelles mobilisées dans la gestion d'environnements liés au vivant à des fins de production (l'irrigation des cultures, la gestion raisonnée des pratiques de désherbage, la conduite de la taille en 
viticulture et en arboriculture) ; - La conception et l'usage de la simulation dans l'enseignement des situations professionnelles ; - La place de l'expérience et de la formation dans la construction et le développement des compétences professionnelles. Ses travaux s'appuient sur les cadres théorique et méthodologique développés en didactique professionnelle.

Adresse : Etablissement National de l'Enseignement Supérieur Agricole de Dijon (ENESAD) Unité propre Développement Professionnel et Formation, Équipe de Didactique Professionnelle Boulevard du Docteur PetitJean

21000 Dijon

\section{Courriel : sylvie.caens@educagri.fr}

Antonietta SPECOGNA est maître de conférence en Psychologie, UP Développement Professionnel et Formation, équipe de Didactique Professionnelle, ENESAD Dijon. L'objet scientifique de ses travaux se centre autour de l'interaction entre au moins deux interlocuteurs au travail. En considérant le langage verbal comme une trace de l'activité des opérateurs en situation de travail elle montre par l'analyse du travail, en faisant émerger les raisonnements cognitifs que les opérateurs mettent en oeuvre aux moments où ils effectuent leurs diverses tâches, comment les compétences développées par les uns et les autres se développent, comment les sujets construisent des relations, comment tout ceci contribue à la conception de formation, au développement du pouvoir d'agir des individus. En somme, elle allie le courant de la psychologie de l'interaction au courant de la didactique professionnelle.

Adresse : Etablissement National de l'Enseignement Supérieur Agricole de Dijon (ENESAD) Unité propre Développement Professionnel et Formation, Équipe de Didactique Professionnelle Boulevard du Docteur PetitJean

21000 Dijon

\section{Courriel : $\underline{\text { antonietta.specogna } @ \text { educagri.fr }}$}

- Ludovic DELÉPINE, chercheur en Intelligence Artificielle, était en poste au Centre National d'Etudes et de Ressources en Technologies Avancées à Dijon (ENESAD-CNERTA) et est aujourd'hui Administrateur en Nouvelles Technologies au Parlement Européen à Bruxelles. Ses activités de recherche concernent la conception et les usages d'outils technologiques. L'originalité de ses travaux sont d'étudier sous un double regard les relations entre l'homme et des outils technologiques : celui de l'informaticien et celui du sémioticien. Sous le regard de l'informaticien, il s'agit de la conception d'expérimentations sur des objets informatiques mettant en œuvre des instruments informatiques réalisés et bien adaptés aux questions posées sur ces objets. Sous le regard du sémioticien, il s'agit de poser les bonnes questions sur l'apparence des objets informatiques pour les utilisateurs et de formuler des recommandations pour une meilleure conception de ces objets informatiques.

Adresse : Centre National d'Etudes et de Ressources en Technologies Avancées (CNERTA) Boulevard du Docteur PetitJean

21000 Dijon

\section{Courriel : $\underline{\text { udovic.delepine } @ \text {,europarl.eu.int }}$}

Stéphane GIRERD, ingénieur d'étude, est responsable de l'équipe de conception de ressources interactives d'Educagri éditions, ENESAD-CNERTA, Dijon. Ses travaux d'étude concernent : - la méthodologie de conception d'outils multimédia pédagogiques (didacticiels, simulateurs, ressources en ligne, ...), et sur l'intégration de l'interactivité en pédagogie ; - l'analyse des usages du multimedia en formation, notamment sur le rapport apprenant-machine dans le cadre d'un apprentissage ; -l'intégration et la pertinence des nouveaux outils dans des dispositifs de formation. Ses réalisations : - conceptionréalisation de cédéroms pédagogiques ("Comprendre le Goût ». Prix Roberval multimédia 2000. Mention spéciale du jury, prix Cervod 1999. "Les manuscrits de Cîteaux », (programme européen 
Info2000. Produit RIP.), « Délos », « Solimage »...) ; - réalisation de films pédagogiques (« entre réel et virtuel » sur la formation à distance, ...) ; - conception-réalisation de DVD pédagogiques (« conversion à l'agriculturebiologique $», \ldots)$.

Adresse : Centre National d'Etudes et de Ressources en Technologies Avancées (CNERTA)

Boulevard du Docteur PetitJean

21000 Dijon

Courriel : stephane.girerd@educagri.fr

\section{Référence de l'article :}

Sylvie CAENS-MARTIN, Antonietta SPECOGNA, Ludovic DELÉPINE, Stéphane GIRERD, Un simulateur pour répondre à des besoins de formation sur la taille de la vigne, Revue STICEF, Volume 11, 2004, Rubrique, ISSN : 1764-7223, mis en ligne le 25/04/2004, http://sticef.org

(C) Revue Sciences et Technologies de l'Information et de la Communication pour l'Éducation et la Formation, 2004 